\title{
Le plurilinguisme dans l'enseigne commerciale: La ville de Louxor comme modèle
}

\author{
Dr. Mohamed Abdelbaki Ahmed Abdelsayed \\ Dr. Mohamed Abdelbaki Ahmed \\ Département de Français - Faculté des Lettres \\ Université du Sud de la Vallée
}

\section{Introduction:}

Une langue est un bon système de signes linguistiques, vocaux, gestuels ou graphiques, qui donne la chance à la communication entre toutes les sociétés en général et les membres de la communauté en particulier. Toutes les sociétés ont un système linguistique propre qui se caractérise par le sens, la forme et le son. De plus, la variation linguistique devient présente pas seulement dans les pays différents linguistiquement mais aussi dans les pays partageant les mêmes idiomes langagiers. La ville de Louxor est l'une des cités les plus touristiques dans le monde avec son accueil d'un grand nombre de touristes chaque année grâce à la présence des monuments antiques et culturels dans la ville. Dans la vie quotidienne nous observons l'échange langagier en langues étrangères d'une manière récurrente et féconde.

Cet échange se manifeste dans les expressions quotidiennes entre les touristes et les guides d'une part et sur les enseignes commerciales d'une autre part. Ce phénomène nous a conduit à étudier plus précisément la situation plurilinguistique en ce qui concerne les enseignes commerciales, qui se distinguent par une forme moderne et écrite en langue arabe et étrangère. Quels sont alors ces critères de l'écriture linguistique de l'enseigne commerciale ? le choix de l'enseigne se fait-il d'une manière logique ou spontanée? L'énonciation est-elle parfaite dans l'enseigne? Le choix des enseignes commerciales est -il de nature à affecter l'importance des ventes et des achats au sein de la ville ? Quelles langues étrangères sont aujourd'hui ancrées dans le marché langagier de Louxor? Quelles langues étrangères sont les plus diffusées dans la ville? Nous tenterons de répondre à ces différentes questions à l'aide d'un questionnaire appliqué et communiqué aux propriétaires de boutiques et aux clients de ces magasins.

\section{Le plurilinguisme et l'espace:}

Selon Caroline Julliard, le terme plurilinguisme désigne «des institutions de contact entre plusieurs langues ou variétés, présentées aussi bien dans les répertoires verbaux que dans la communication sociale ${ }^{1} \gg$. De cette définition, nous pouvons comprendre que « la ville» tient une place très privilégiée pour la raison de l'existence même du plurilinguisme. Reconnaissons que la spécificité de ce type de terrain réside dans le fait que les formes et les fonctions des différentes langues varient au rythme des changements sociaux qui résultent des faits urbains. La ville se définissant comme «une somme de lieux et d'espaces, le lieu serait de l'ordre de l'immuable, de l'être la, repères visuels, lieux commémoratifs, monuments, 
magasins. L'espace serait pour ainsi dire, façonné par les actions, les mouvements, les visions des habitants ou des passants ${ }^{2} \gg$.

Les différentes représentations de la ville qui se construisent par la mise en mots des lieux, des espaces et les frontières de l'urbanisation se définissent ainsi à travers des territoires sociaux et langagiers. La ville apparait dans un même temps comme un point de convergence des migrations et de leurs langues et comme un carrefour linguistique par excellence comme le dit Calvet dans son livre intitule les voix de la ville:

"Telle est une pompe, la ville aspire du plurilinguisme et recrache $d u$ monolinguisme, et elle joue ainsi un rôle fondamental dans l'avenir linguistique de la région ou de L'Etat ${ }^{3 " .}$.

Nous pouvons ainsi constater que le facteur urbain a indiscutablement quelque effet sur les langues et les représentations linguistiques qui accordent une place assez considérable à la mobilité spatiale induite par les migrations ou déplacements d'un pays à un autre, différents les uns des autres par des idiomes linguistiques.

De ce qui précède, nous estimons que du fait d'une concentration progressive de population d'origines variées, un plurilinguisme de contact se développe et se transmet en ville. Cependant, afin que la description du plurilinguisme urbain soit parfaite, il y a certes des paramètres explicatifs dont on doit tenir compte. Il s'agit notamment de :

- La dimension urbaine (taille, nombre et diversité des habitants, nombre et diversité des langues et variétés).

- La relation entre la ville et son environnement (proche ou moins proche: monde rural, autres villes, région, pays dans son entier, voire pays voisins ou pays colonisateurs)

- La composition des quartiers et leur différenciation.

- la dimension du coin où l'on vit et celles des espaces de sociabilité ouverte

- le rôle des statistiques démographiques et économiques.

- il faut compter sur le savoir partagé des citadins en cas de l'absence de certains paramètres ${ }^{4}$.

\section{L'enseigne commerciale et la ville}

Selon le Grand dictionnaire universel du XIXe siècle, l'Enseigne se définit ainsi:

S.f "du latin Insigna, pluriel du neutre insigne, qui est forme de in,en et de signium, en premier lieu, signe, marque distinctive puis indice d'identité, d'authenticité, de vérité (...) c'est ainsi, une indication que l'on met au dessus de l'entrée d'un établissement commercial, pour indiquer la nature du commerce et souvent le nom du commerçant ${ }^{5}$.

Par ailleurs, dans son article intitulé les enseignes, leur origine et leur rôle, Emile Ginot, définit l'enseigne comme suit :

"Nous définirons d'abord l'enseigne, d'après son sens étymologique, signum, un signe quelconque destiné à distinguer un objet entre plusieurs autres de même nature ${ }^{6 "}$, et il reprend "ou, pour parler avec Ménage une marque particulière qui, aidant à discerner quelques personnes ou quelque chose, la fait connaître ${ }^{7 "}$.

Pour le dictionnaire le Petit Robert du XXe siècle, l'enseigne est désigné comme étant un : "Panneau portant un emblème, une inscription, un objet 
symbolique, qu'un commerçant, un artisan met à son établissement pour se signaler au public $8 . "$

Nous comprenons à travers ces différentes définitions que l'enseigne est une marque ou une inscription qui sert à indiquer la présence de tel ou tel commerce. Elle garantit une relation intime entre vendeur et acheteur et se présente généralement comme un panneau portant l'emblème ou un objet symbolique qu'un commerçant ou un artisan accroche pour la visibilité de son magasin ou à son institution. Elle est un véritable moyen pour attirer et informer les clients.

Signalons que la ville est perçue comme une entité urbaine multidimensionnelle. Elle témoigne des hommes qui s'inscrivent dans différentes langues. Elle regroupe de traces d'identités et de langues multiples ${ }^{9}$. Elle se donne à voir et à lire dans toute la diversité qui la réagit. Les enseignes commerciales comportent bien des langues et parfois des signes se référant à la réalité du milieu dans lequel elles sont inscrites. Autrement dit, les enseignes commerciales peuvent être tenues comme une façon particulière d'énoncer des discours, de communiquer et de dire ce qui caractérise le commerçant ou le type d'activité qu'il pratique. Ces enseignes représentent pour ainsi dire, une image visuelle du langage social de la communauté linguistique qui parle et qui entonne ce type du discours dans la vie quotidienne comme le dit Agnès Millet:

"La ville semble le lieu d'une communication sans fin ou l'écriture et le dessin se réinventent sans cesse en figures pour mieux dire son appartenance et sa différence. La ville se fait support multiple de ce que l'on pourrait appeler (...) un langage visuel ${ }^{10 " .}$.

Nous pouvons dire que les inscriptions sur les enseignes des magasins est un type du discours qui dure et se veut être continu à travers le temps. Une sorte de singularité individuelle ou commerciale et aussi une forme d'une appartenance à un groupe élitiste. Pour cela les enseignes commerciales deviennent un symbole du parler quotidien de la communauté linguistique comme le signale Zohra Benmansour dans son étude sur le marquage signalétique en Algérie:

"Dans le milieu urbain, les enseignes de magasins qui se donnent à voir dans les différentes rues de la ville se veulent révélatrices de la société urbaine dans la mesure ou elles répondent particulièrement à des caractéristiques d'ordre économique, identitaire, culturel et linguistique des commerçants ou des locuteurs de cette ville ${ }^{11}$."

D'ailleurs, dans la ville il y a deux types d'écrits: officiels et individuels. Les premiers sont appelés des écrits in vitro, selon les termes de Calvet, qui sont diffusés par des institutions et sont caractérisés par le cachet formel et légal de la part de l'état. Ces écrits ont une tendance unificatrice à tous les niveaux en remplissant une fonction essentiellement d'orientation ou d'information. C'est une sorte de mise en ordre de l'espace urbain. Nous rencontrons ces écritures sur les plaques et les panneaux de signalisation dans la ville ou à vrai dire sur toutes les affiches formelles indiquant les différentes directions éventuelles possible dans une ville.

Par contre, à travers les écrits individuels ou in vivo, selon Calvet, les individus essayent d'affirmer leur présence et leur appartenance à un groupe ou à une société entière par l'appropriation d'un ou plusieurs systèmes d'écritures. Leur présence sur les enseignes des magasins ou autres, ne soumet pas ceux-ci à une loi 
puriste ou académique. Par conséquent ils subissent beaucoup de variation sur le niveau sémantique et lexical des termes et des expressions.

\section{La ville de Louxor}

Comme nous le savons bien, Louxor est une ville située sur la rive droite du Nil, en Haute Égypte, à environ $700 \mathrm{~km}$ au sud du Caire et environ $300 \mathrm{~km} \mathrm{au}$ nord d'Assouan. Un grand nombre d'habitants vivent directement ou indirectement du tourisme.

Le site de Louxor qui accueille plus de quatre millions de visiteurs par an, est l'un des endroits les plus touristiques de l'Égypte et constitue la partie sud de l'ancienne Thèbes. Faisons remarquer que le tourisme représente une ressource par excellence de la ville de Louxor et par conséquent, la présence des langues étrangères reste aussi féconde dans les conversations que dans les enseignes des magasins, des hôtels et des bureaux touristiques. Ici, nous nous intéressons exclusivement aux enseignes des magasins puisqu'elles représentent une image visuelle du plurilinguisme dans la ville.

D'autre part nous pouvons dire que nous nous intéressons aux écrits des individus "in vivo" c'est-à-dire les écrits qui se caractérisent par la variété langagière. Pour les écrits "in vitro", nous avons remarqué qu'ils sont généralement en langue officielle c'est-à-dire l'arabe classique. Pour cette raison, nous avons opté pour les écrits individuels puisqu'ils sont exprimés en différentes langues et l'emploi du plurilinguisme y reste croissant. Ces écrits freinent la politique linguistique ${ }^{12}$ de l'Etat, stimulant à l'emploi de la langue arabe dans tous les contextes formels et aussi l'enseignement des langues étrangères dans les méthodes éducatives comme langue secondaire comme stipulé dans le deuxième article de la constitution égyptienne:

"L'islam est la religion de l'Etat, la langue arabe est la langue officielle et les principes de l'islam sont la source essentielle de la législation ${ }^{13}$."

Donc nous comprenons que l'arabe devient la langue officielle de l'Etat et que le choix des langues étrangères à la place de l'arabe quant aux inscriptions sur les enseignes des magasins revient au seul bon vouloir des personnes et non aux règlements de l'Etat.

Pour mener à bien notre recherche nous avons d'abord commencé par photographier un corpus qui dépend des noms des commerces. Ce corpus renferme des enseignes écrites en langues différentes: arabe, français, anglais, et autres langues.

Notre démarche était participative à travers la distribution de questionnaires appliqués aux commerçants et aux clients afin d'évaluer le regard de chacun sur l'enseigne écrite et pour mieux délimiter les particularités plurilinguistiques de l'enseigne commerciale en général et dans la ville de Louxor en particulier.

\section{Analyse des enseignes commerciales}

Notre analyse des enseignes commerciales s'est fait suivant deux techniques de classification.

- La première est d'ordre intrinsèque et consiste à mettre l'accent sur l'aspect interne des enseignes qui s'intéressent à l'étude syntaxique et lexical des mots composant ces enseignes. 
- La deuxième est de nature extrinsèque. Elle s'attèle à montrer l'aspect externe des enseignes qui se donnent à voir sous telle ou telle forme et dans telle ou telle langue. Nous pouvons dire que les discours véhiculés par ces écrits représentent d'une manière originale la réalité urbaine de cette ville dans toutes les dimensions sociolinguistique, historique, économique, politique et culturelle. Il en ressort que l'analyse linguistique de ces devantures de magasins peut non seulement montrer des spécificités plurilinguistiques, spatiales et identitaires de la ville de Louxor mais aussi, permettre de connaître les critères du choix langagier de l'enseigne écrite et exprimée en langues différentes.

\section{Les fonctions de l'enseigne commerciale}

Nous pouvons dire que l'enseigne commerciale se caractérise par quelques particularités spécifiques. L'enseigne en général remplit deux fonctions l'information et l'ostentation. L'enseigne donne le nom du commerce c'est-à-dire la dénomination commerciale pour que le passant puisse individualiser facilement le commerce. Voici un exemple:

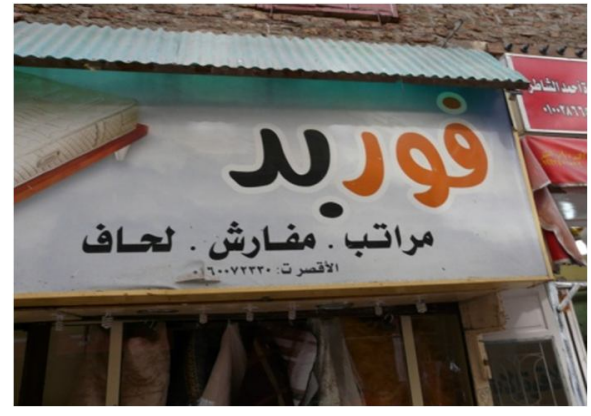

Rue Manchia

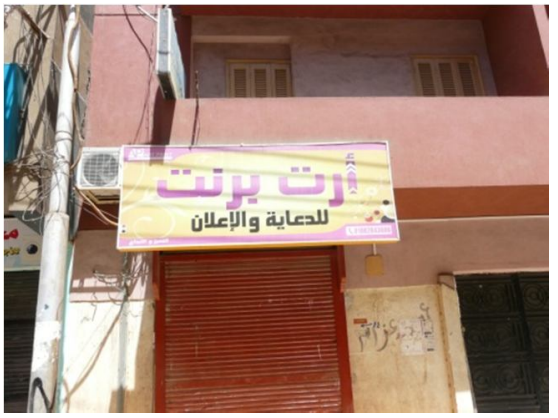

Place de la gare

Dans ces deux enseignes, nous remarquons le nom du magasin puis suivi le type de l'activité commerciale, celle- ci est une sorte d'individualisation par rapport aux autres commerces de mêmes activités dans la ville ou dans le même centre commercial.

\section{L'enseigne, un phénomène publicitaire}

L'enseigne commerciale est ostentatoire spécialement celle iconique. Cela se représente par la présence des éléments lumineux et les jeux de couleurs. Ces éclairages invitent le passant ou le client à regarder, à lire et à créer involontairement chez lui le besoin et l'envie de lire puis entrer dans le magasin pour voir sur place le type et la qualité du produit écrit ou lumineux. Cette ostentation confère à l'enseigne, la qualité de servir de moyen publicitaire comme le dit Verdier dans son livre consacré à l'étude de la publicité:

"L'enseigne lumineuse est exclusivement une publicité de notoriété(...) Elle fait la publicité du détaillent plutôt que de celle du ou des produits ${ }^{14}$."

Dans notre analyse des enseignes commerciales dans la ville de Louxor, nous avons observé que le commerçant cherche par tous les moyens à attirer son client par le choix des mots simples et déchiffrables comme l'indique l'exemple suivant: 


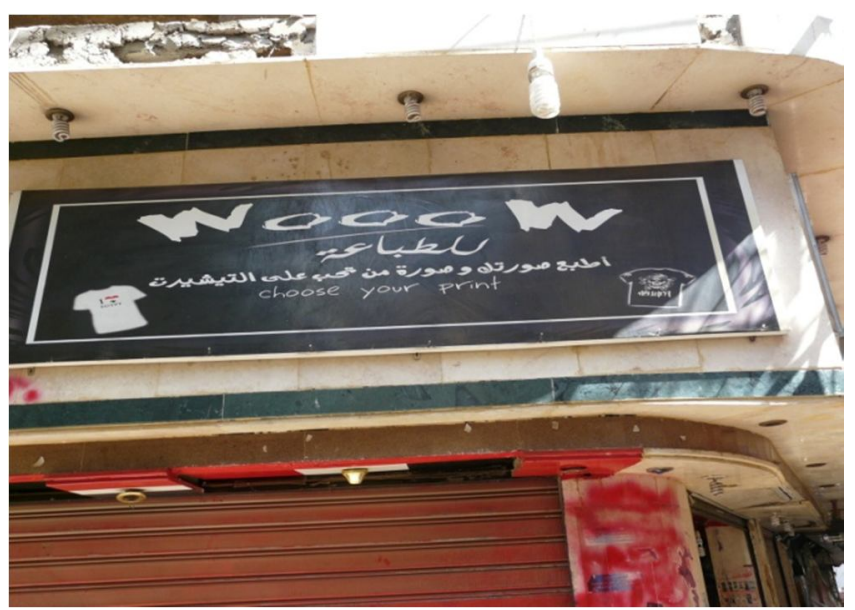

Rue Salah Eldin

Dans le titre de l'enseigne ci-dessus, le commerçant tente d'exercer une action psychologique sur le public ou bien sur les passants. Nous observons que le titre attire l'attention WOOOW puis une phrase encourageant le public à venir vite et nombreux chez lui pour découvrir l'esthétique et l'art du commerçant. Donc avec ce style publicitaire, le commerçant n'a aucune finalité que d'attirer le client et vendre ses produits à travers l'emploi de toutes les structures grammaticales et sémantiques possibles.

\section{- L'enseigne, un phénomène culturel}

Nous pouvons dire que l'enseigne est considéré comme un code qui renvoie à certaines réalités socioculturelles. L'écrit de l'enseigne permet de montrer l'idéologie du propriétaire du magasin. Dans ce cas l'enseigne vise un client propre. Voici un exemple:

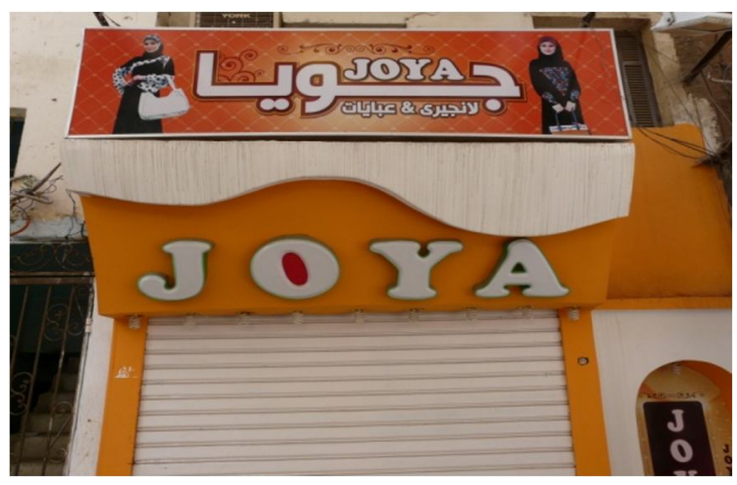

Place de la gare

Dans la photo ci-dessus le commerçant s'adresse aux femmes qui veulent ou cherchent à porter des vêtements orientaux. Son choix pour le nom Joya, un nom venu du bas latin qui signifie joyaux, c'est pour attirer l'attention mais la photo de la femme à côté de l'enseigne et les mots écrits sous le nom signifient que le propriétaire cherche à attirer les femmes en précisant la nature des activités aussi bien par les mots que par la forme. 
Parfois nous rencontrons dans la ville de Louxor des enseignes commerciales écrites en langues étrangères qui n'ont aucune finalité que de donner l'impression au client que c'est un magasin moderne ou à la mode. Le commerçant fait alors usage dans ce cas, des mots quasiment mal perçus par la majorité des clients ou des passants, hormis ceux qui comprennent l'italien, comme nous le voyons dans cet exemple:

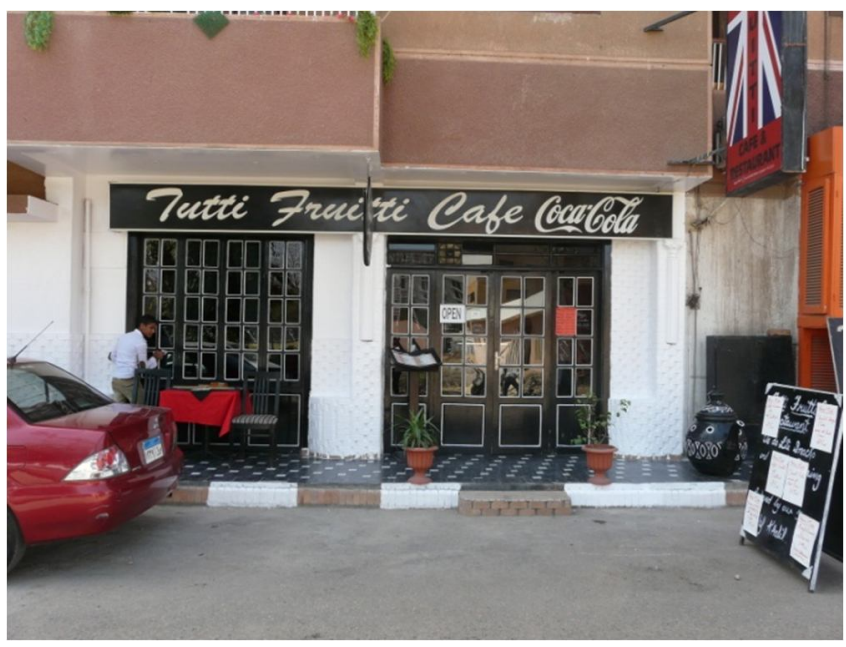

Rue Ramsès

Dans la photo ci-dessus, l'enseigne est bilingue, puisque nous trouvons des mots italiens comme Tutti Fruit, puis le mot français Café qui indique l'activité du magasin. Cela traduit que le propriétaire du magasin cherche à attirer le client chez lui soit par l'emploi des mots étrangers, en suscitant la curiosité de découvrir des goûts nouveaux, soit simplement par la manière italique de l'écriture. Ceci dénote que le propriétaire ne vise en réalité pas les clients italiens mais simplement tous les clients potentiels friands de la modernité ou du prestige.

\section{L'enseigne commerciale comme système d'énonciation}

Selon P.Charaudeau, on peut définir l'énonciation comme l'acte individuel de l'emploi de la langue pour l'opposer à l'énoncé qui est un objet linguistique résultant de cette utilisation ${ }^{15}$.

"Dans toute pratique linguistique il y a énonciation. L'énonciation constitue le pivot de la relation entre la langue et le monde; d'un côté, elle permet de représenter dans l'énoncé des faits, mais d'un autre côté, elle constitue elle-même un fait, un événement unique défini dans le temps et dans l'espace ${ }^{16}$."

Il apparaît que la communication humaine exige une présence de différents participants qui se nomment interlocuteurs et elle doit être organisée selon le milieu, la culture et le temps. Les écrivains et les affichistes adoptent un code propre qui devient à la portée d'une grande partie de lecteurs ou de passants.

D'ailleurs nous pouvons dire que l'enseigne commerciale est un texte ou une œuvre littéraire comme le note H. Verdier:

"L'ouvre vise un public. Cela veut dire que l'écrit sollicite une lecture (...) et que l'artiste qui produit l'œuvre s'adresse à un public qui rependra à cette sollicitation ${ }^{17}$." Par conséquent nous pouvons déduire que l'écrit de l'enseigne est 
considéré comme un système d'énonciation par excellence parce qu'elles sont faites par un réalisateur ( le commerçant), destinées à un public bien déterminé (clients ou passants) et dans un contexte bien précis ( le milieu urbain). Voici un exemple qui illustre cette idée:

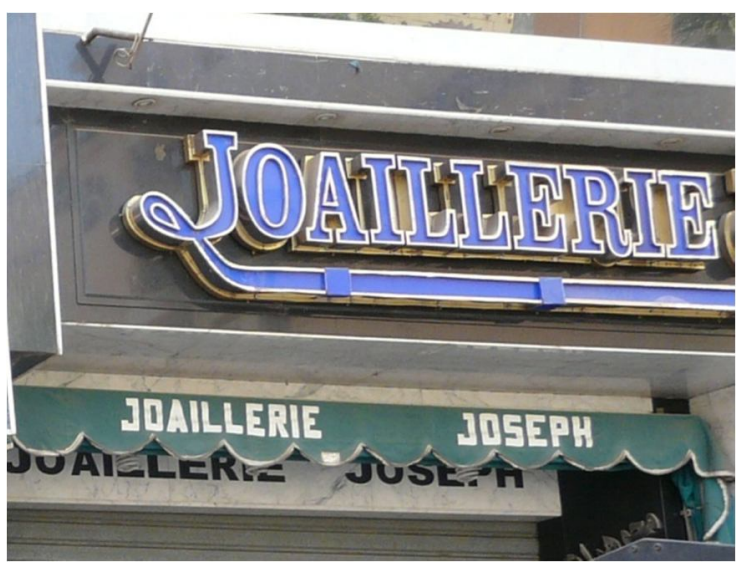

Dans l'exemple ci-dessus,

\section{Rue de la Poste}

- l'énonciateur est le propriétaire du magasin qui s'appelle Joseph,

- le récepteur est le passant ou le client qui connaît le français,

- le message c'est la vente de Joaillerie,

- le code est écrit en langue française.

- Le but c'est d'attirer le public à acheter ses produits ou ses ventes.

Ici, la situation de communication est correcte puisque ses éléments sont disponibles, mais si le passant ne comprend pas le titre du magasin, il sombrera dans l'embarras et se verra obligé de visiter le magasin sur place afin de sonder et d appréhender la véritable nature de l'activité pratiquée.

Nous pouvons schématiser cette situation de communication dans l'enseigne par la figure suivante:

\section{Le contexte}

Joaillerie Joseph (enseigne commerciale à la ville de louxor)

Le destinateur

Commerçant le message

titre du magasin

\section{Le code}

La langue française le destinataire

Le passant/ le client

\section{Canal}

La luminosité et les lettres en majuscule

Faisons remarquer ici que la situation d'énonciation se construit justement à travers l'espace situé et façonné par le magasin lui-même. Les magasins environnants, les passants dans toutes les formes interactionnelles, les marques 
urbaines, l'habitat, les circulations et mouvements, etc. Bref "c'est dans cet espace de monstration mais aussi représenté que les enseignes prennent tout leur sens et toute leur valeur ${ }^{18}$."

Parfois on assiste à une situation de communication incompréhensible et presque codée dans l'enseigne commerciale. Voici un exemple:

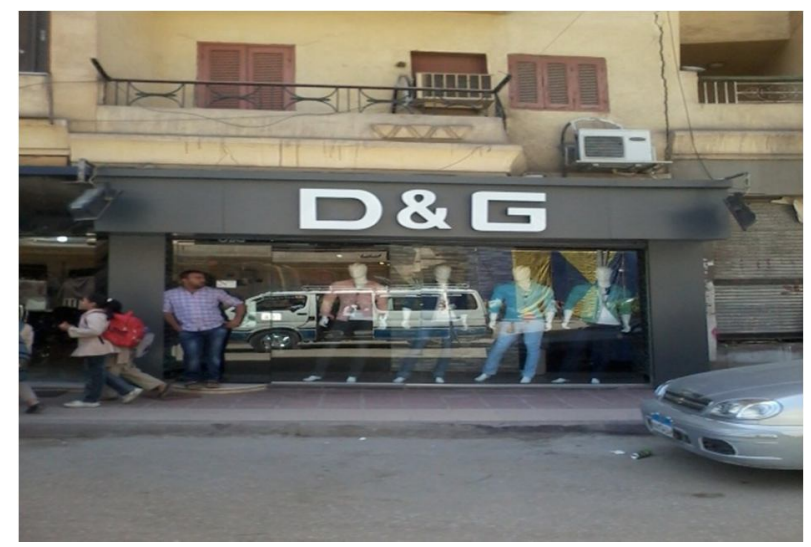

Rue Isis

Dans l'enseigne ci-dessus nous ne pouvons préciser ni le nom du propriétaire du magasin ni la nature de l'activité commerciale pratiquée. Ici le locuteur joue sur la langue des sigles mais malheureusement le récepteur ne la comprend qu'en fréquentant le magasin. Ce processus consiste à attirer l'attention du client ou du passant et ensuite, le laisser se poser des questions sur le type des activités. Nous pouvons dire que l'emploi du sigle est un phénomène langagier qui se trouve aussi bien dans le code écrit que dans le code oral. Les sigles répondent à l'économie du langage et permettent d'éviter l'écriture de longs mots, de syntagmes ou de longues phrases. Mais dans l'exemple cité le sigle ne fait que compliquer le sens de l'enseigne et on est obligé de fréquenter le magasin pour la décrypter. Voici un autre exemple pour mieux comprendre cette idée:

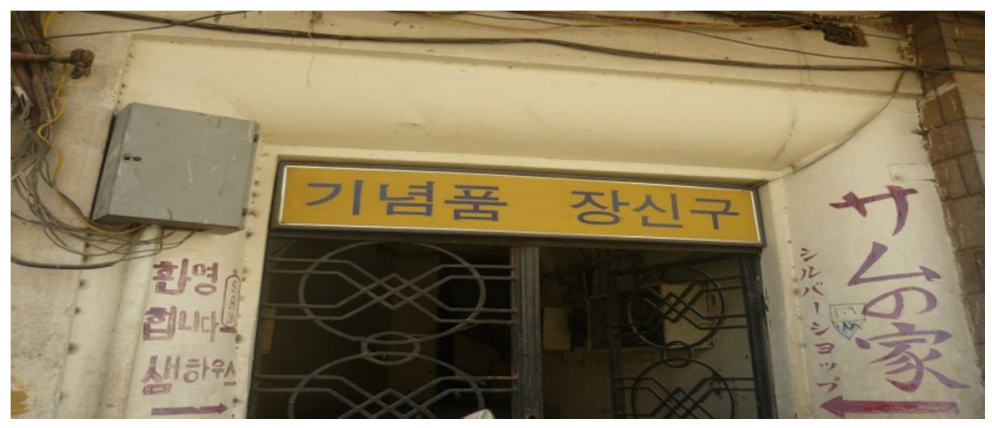

Rue du marché touristique

Dans cet exemple, le locuteur vise un public particulier puisque les autochtones de Louxor n'emploient pas le coréen dans la vie quotidienne et aussi c'est une langue rare même au sein de cette ville touristique. Donc c'est une situation 
de communication parfaitement codée, un dialogue des sourds entre le locuteur et le passant à l'exception de personnes spécifiques, maitrisant parfaitement cette langue.

En me renseignant auprès d'un collègue du département de la langue coréenne de l'université d'Ain Chams au Caire, j'ai compris le sens de cette enseigne : c'est un magasin de bijouterie et de souvenirs touristiques. Il apparait clairement que c'est le touriste coréen qui est automatiquement ciblé. Les autres nationalités sont pour ainsi dire, exclues de la situation de l'énonciation.

Nous observons également dans cet exemple que la valeur référentielle donnée à voir se trouve imposée sans modalisation et sans négociation et la situation de l'énonciation devient asymétrique puisque les récepteurs n'ont pas le droit de la prise de la parole comme le dit Kerbat Orecchioni : "si les destinataires, les passants, les clients sont bien visés par le locuteur, ils restent non loquant dans un acte de communication unilatéral ${ }^{19}$." Donc le commerçant peut offrir ou imposer dans l'espace public, à travers l'enseigne du magasin, son univers de référence culturel, personnel et intime et c'est une manière de l'appropriation de l'espace par son imaginaire propre et le client doit imaginer le sens d'une manière fantasmée.

\section{La forme nominale des enseignes}

La forme des enseignes des magasins renseigne le plus souvent sur la nature de l'activité du magasin, le nom de la personne exerçant cette activité et le nom que porte le lieu de l'activité en question. D'après la connotation que nous avons faite sur les enseignes des magasins, nous avons remarqué que la plupart d'entre elles sont écrites sous forme de noms. Voici des exemples:

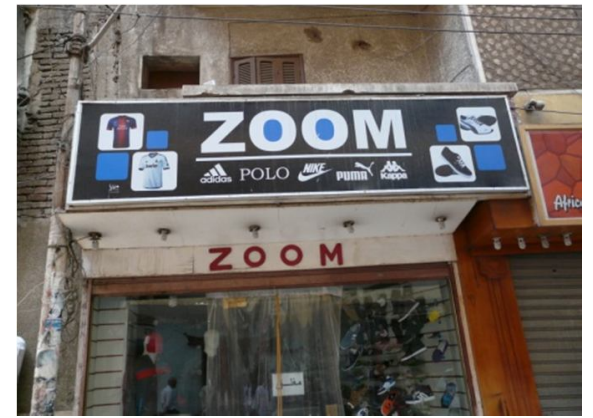

Rue de la Television

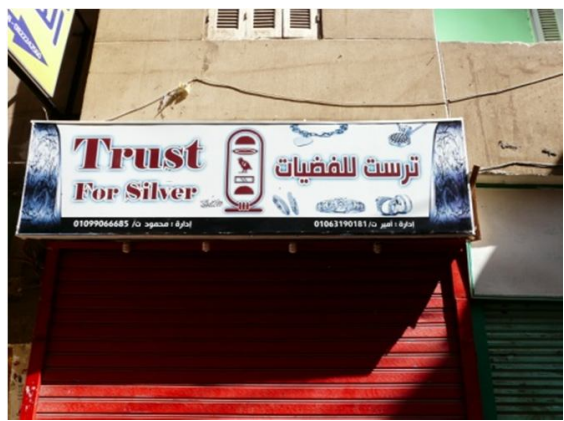

Rue Nazlet Elzanaty

$\mathrm{Au}$ niveau des deux enseignes ci-dessus, nous remarquons l'absence du verbe. La valeur devient par conséquent intemporelle. Le locuteur emploie le nom sans aucun accompagnement du verbe pour rendre l'information valable et durable à jamais :

"En l'absence du verbe dans la phrase nominale, l'assertion est intemporelle et fait le référant dans une valeur immuable, donnée comme vérité générale voire absolue. Pourtant, la portée référentielle de la phrase, et donc le sens qui en découle, varient selon la construction même de la forme nominale et selon l'évocation du contexte d'énonciation20." 
Nous savons bien également que parmi les valeurs de la phrase nominale, existe l'information succincte et immédiate. Le commerçant, à travers ces courtes phrases, a comme objectif d'accrocher ses clients et même les passants et de les inviter implicitement à fréquenter les magasins en les informant rapidement. Néanmoins ces expressions nominales varient d'un magasin à l'autre et n'ont pas les mêmes formes de constructions. Dans ce qui suit nous verrons comment l'enseigne peut renvoyer à l'énonciateur en portant le nom de son réalisateur; le commerçant ou bien elle peut renvoyer à l'espace ou la rue dans lesquels elle prend existence.

\section{La référence dénominative ou l'indexalisation de la personne}

Selon M. Grevisse dans Le bon usage, le nom propre ne possède pas de sens véritable, et il peut se rattacher à ce qu'il désigne par un rapport qui n'est pas sémantique, mais par une convention qui lui est propre ${ }^{21}$. Nous pouvons dire que le nom propre et plus particulièrement le nom de la famille inscrit la personne dans sa filiation, ancré dans un passé qui la porte vers l'avenir. Le nom propre sert à identifier, à repérer, à donner existence puisqu'il ne renvoie pas à un sens mais simplement à sa présence sur le marché commercial par son propre nom qui représente sa réputation, son passé et son avenir. Au niveau des enseignes commerciales de la ville de Louxor, nous observons l'emploi multiple du nom de personne ou du patronyme ${ }^{22}$. Voici des exemples:

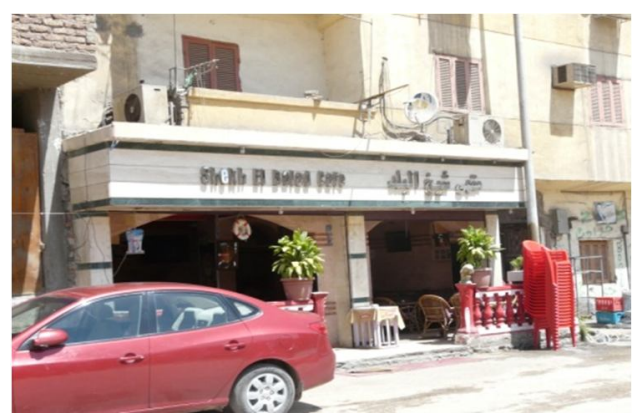

Rue Manchia

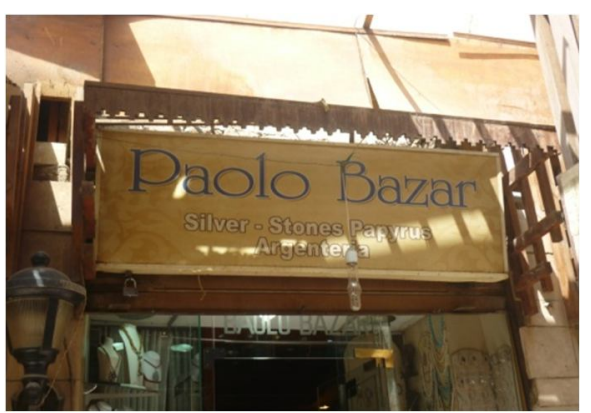

Rue du marché touristique

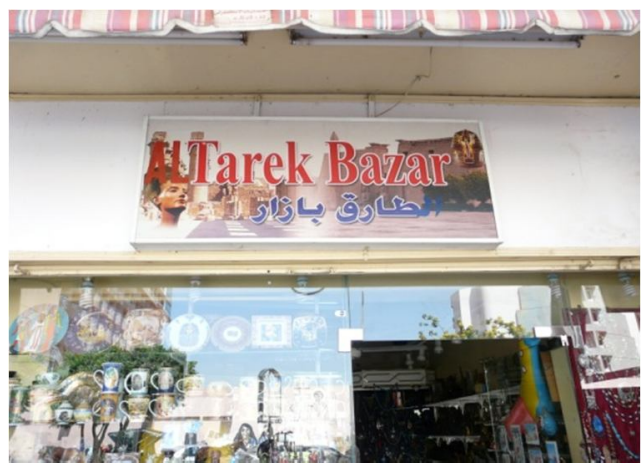

Rue Isis

Concernant les enseignes ci-dessus, nous remarquons que les commerçants en tant que sujets d'énonciation tentent de s'identifier et s'imposer dans l'espace et 
même dans l'imaginaire des clients par l'écriture du nom propre ou le patronyme. Dans ce cas le patronyme engage la parole du sujet comme il "engage son existence en lui donnant un sens, une orientation ${ }^{23}$." Ici le nom propre sert à établir une relation profonde et directe entre le commerçant et son client et lui facilite l'orientation dans l'espace urbain. Au niveau de l'enseigne Cheick Elbalad café par exemple, nous pouvons dire que le Cheikh Elbalad est un personnage responsable du village égyptien. Il est comme le collaborateur du maire au sein du même village et incarne la sagesse et la justice. Le choix de ce nom sur une enseigne commerciale au niveau d'un café symbolise l'authenticité et l'originalité de la place qui est réservée, selon le locuteur, aux hommes sages et sociables ou à toute personne ayant une bonne moralité. Nous pouvons ainsi confirmer que le nom propre et le prénom, en lien avec le référent, sont porteurs de connotation, d'exotisme, d'étrangeté, et de modernité. Même s'il serait populaire ou ancien, il crée une sorte de conformité ou de correspondance avec un ensemble de représentations partagées par un groupe social.

Ainsi, il en résulte de l'analyse des enseignes dans la ville de Louxor, la rareté ou la quasi inexistence de l'emploi du nom propre féminin. Cela fait ressortir la nature virile ou masculine de la profession et de la société, mais cela n'empêche pas de trouver quelques traces:

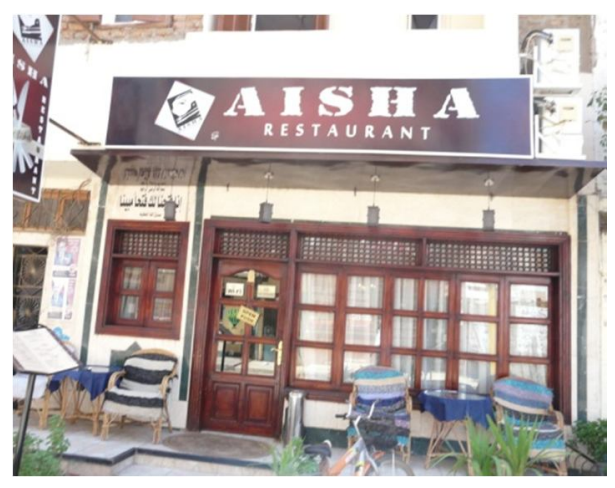

Rue Alfayrouz

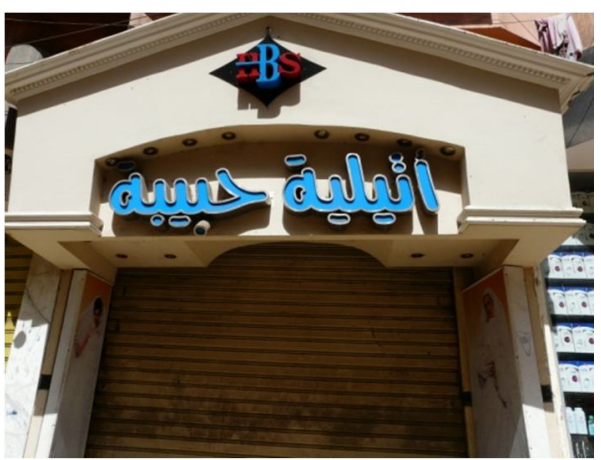

Rue de la Corniche

Suivant le questionnaire appliqué aux locuteurs des magasins, il apparait que le locuteur puisse choisir le prénom de sa fille par exemple ou un nom cher à lui comme c'est le cas au niveau de la première enseigne. Au niveau de la deuxième enseigne, il s'agit du prénom de la locutrice. Ce qui apparait tout à fait normal puisque souvent, on rencontre à Louxor ou en Egypte en général, que les commerces de vente de vêtements ou d'accessoires féminines sont souvent tenus par les femmes. Ceci nous pousse à déduire que le choix de l'enseigne revient à la nature de l'activité commerciale pratiquée dans le magasin.

D'une part, nous constatons que les formulations du texte au niveau des enseignes des magasins, se présentent sans aucun verbe. L'absence de celui-ci n'implique cependant pas l'absence de prédicat qui sera assuré par l'un de deux termes. Nous observons que les constructions nominales ont deux éléments, les deux 
parties ne sont reliées par aucun élément grammatical; elles deviennent juxtaposées et entretiennent une relation prédicatives. Voici un exemple:

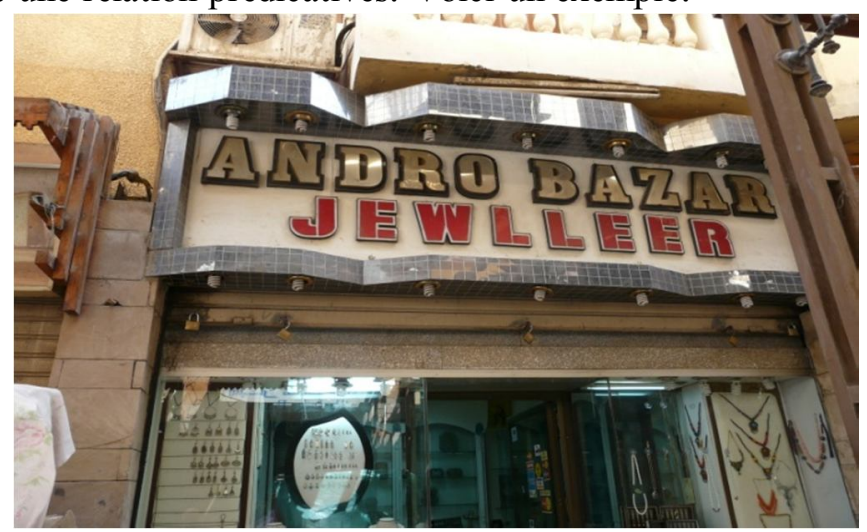

Rue du marché touristique

D'autre part, au niveau des devantures des magasins du type dénominatif, nous observons que le nom propre peut être antéposé ou postposé. A Louxor nous comptons beaucoup de noms propres antéposés. Voici des exemples:

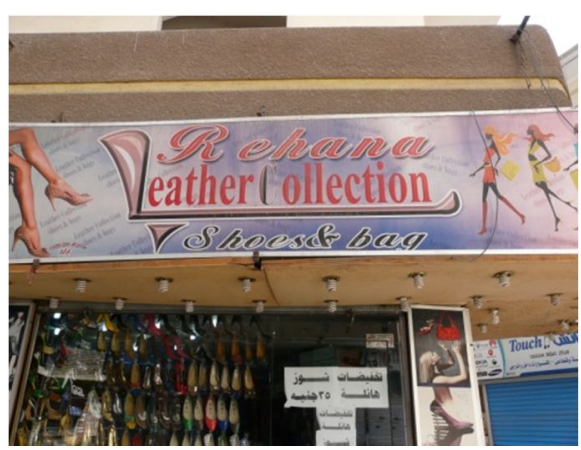

Rue Salah Eldin

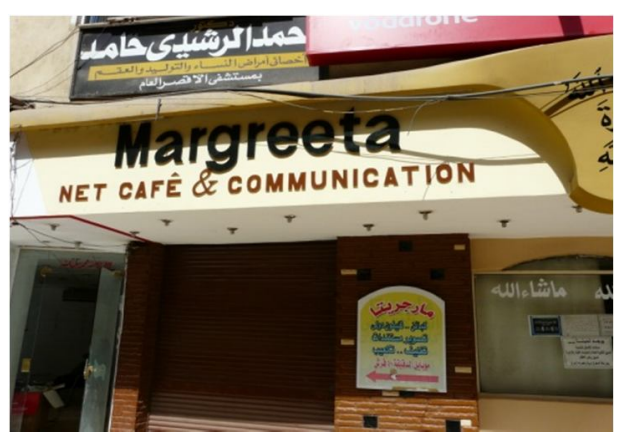

Rue Abou Elgoud

Cette antéposition des noms propres dans les enseignes prend une valeur de reconnaissance sociale et de distinction spatiale. Cette technique de la mise en avant traduit effectivement la volonté du commerçant à mettre en valeur son magasin et le rendre exceptionnel par rapport à d'autres magasins du voisinage. Pour cela on voit parfois chez quelques commerçants l'écriture du nom propre en majuscule ou en lettres colorées pour attirer l'attention du passant. C'est donc un signe d'appropriation dans l'espace et un moyen pour s'imposer dans lé marché commercial de son quartier ou de son environnement.

Contrairement à cette technique de la mise en avant du nom propre, nous constatons une deuxième structure. C'est le cas des noms postposés, c'est-à-dire des noms qui se placent après la dénomination du magasin. Dans ce cas, les enseignes mettent l'accent sur le rôle et la fonction du magasin. Nous trouvons beaucoup de ce type d'enseignes dans notre corpus. Voici des exemples: 


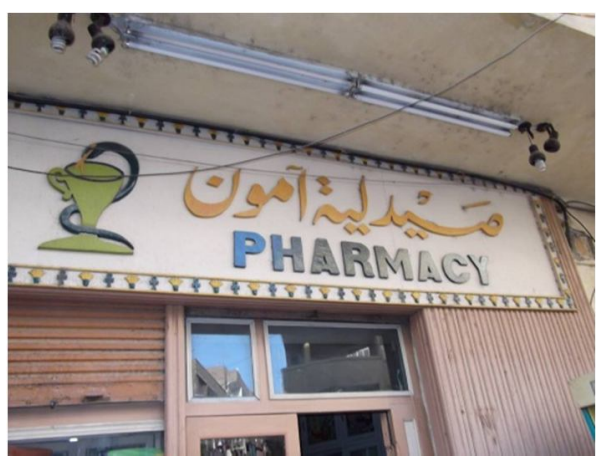

Rue Isis

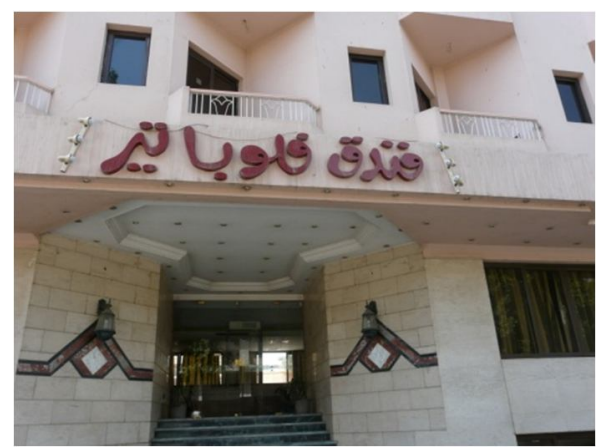

Place du roi Abdallah

Ces occurrences des noms postposés évoquent la forme traditionnelle de l'enseigne et reflète explicitement la nature de l'activité commerciale. Les commerçants, à travers ce type d'enseigne, ne veulent pas s'identifier ou s'imposer dans l'espace, mais ils déclarent uniquement leur présence dans le quartier en écrivant tout directement la fonction du magasin au début de l'enseigne, contrairement aux enseignes antéposées où nous pouvons dire que les commerçants veulent paraître distingués socialement et spatialement par la mise en exergue de leur appartenance identitaire.

\section{- Les formes autodésignantes du magasin}

En ce qui concerne ce type d'enseignes, le nom se concentre sur l'activité commerciale dans le magasin. L'enseigne renvoie au magasin lui-même et devient donc autodésignante. Dans ce cas, le commerçant se met à distance car il ne fait pas paraître son nom sur l'enseigne. Nous rencontrons beaucoup de ce type dans la ville de Louxor et spécialement dans les rues touristiques:

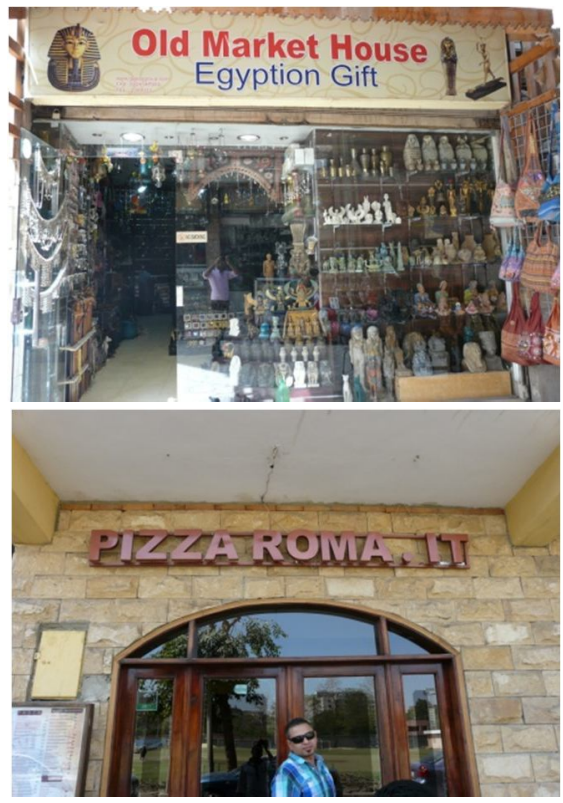

Rue Isis
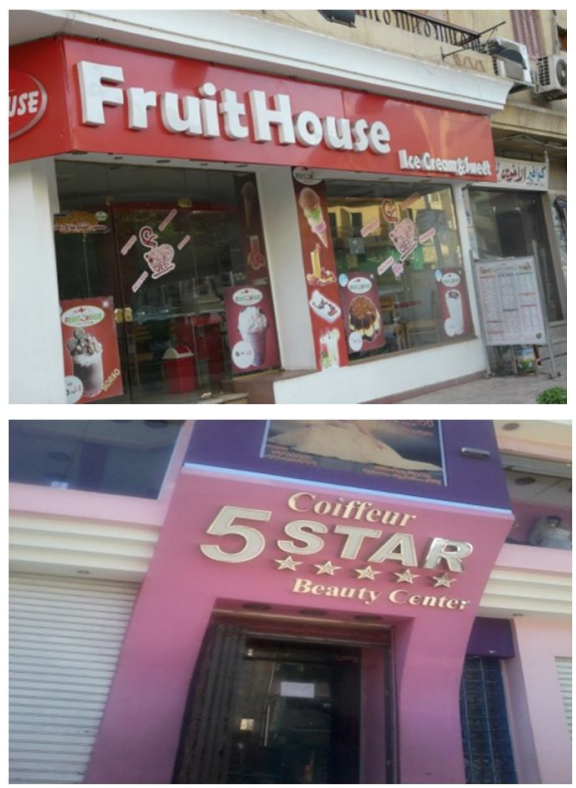

Rue Nazlet Elzanaty 
A la lecture des ces enseignes, nous constatons qu'elles portent des formes simples et elles renvoient généralement à l'espace et elles informent sur la nature de l'activité commerciale d'une manière lisible et compréhensible sans aucune médiation de la part du commerçant ou d'un autre passant dans la rue. On y utilise la forme nominale soit en langues étrangères, soit en arabe. C'est alors un bon moyen d'information du passant sur le magasin d'une manière explicite et directe. Dans ce cas le locuteur n'est pas à la recherche d'une quête identificatoire mais informative comme le dit Romain Lajarge et Claudine Moise:

"L'emploi du caractère générique du magasin sans évocation spécifique a valeur essentiellement informative; présente sans recherche typographique, il ne joue pas sur le particularisme, l'attrait singulier, l'exception commerciale ou l'identification du commerçant 24 ".

Nous observons également, au niveau des devantures des magasins, certaines fautes orthographiques comme ce qui parait dans l'enseigne Old Market House Egyption Gift. Les fautes orthographiques, au niveau des enseignes, signifient que le commerçant et celui qui a rédigé l'enseigne ont un niveau faible en langue étrangère et que le plus important chez eux est de satisfaire coûte que coûte le sentiment d'écrire en langues étrangères pour capter l'attention. Selon ces commerçants, c'est un signe de distinction des autres commerçants.

Nous constatons également une sorte d'exagération dans l'emploi de l'image comme la luminosité croissante. C'est un moyen pour les rendre exceptionnels et attirer les regards des clients et des usagers de ces espaces urbains. Par la mise en valeur de leurs magasins, ils visent également à s'approprier cet espace urbain d'une manière encore plus différente.

\section{La référence spatiale ou l'indexicalisation du ici / la- bas:}

Selon Maingueneau, le point de repère des déictiques spatiaux, c'est "la position qu'occupe le corps de l'énonciateur lors de son acte de communication ${ }^{25} . "$ Maingueneau distingue plusieurs types de ces déictiques comme les démonstratifs, les présentatifs, les éléments adverbiaux26 ... Au niveau des enseignes que nous avons pu recueillir, nous avons constaté des indices spatiaux renvoyant soit au quartier dans lequel elles existent ou bien à un espace géographiquement et culturellement différent et lointain. Parmi ces indices spatiaux, nous pouvons distinguer "ici" et "ailleurs". Nous commençons par les enseignes comportant ici. Voici des exemples: 

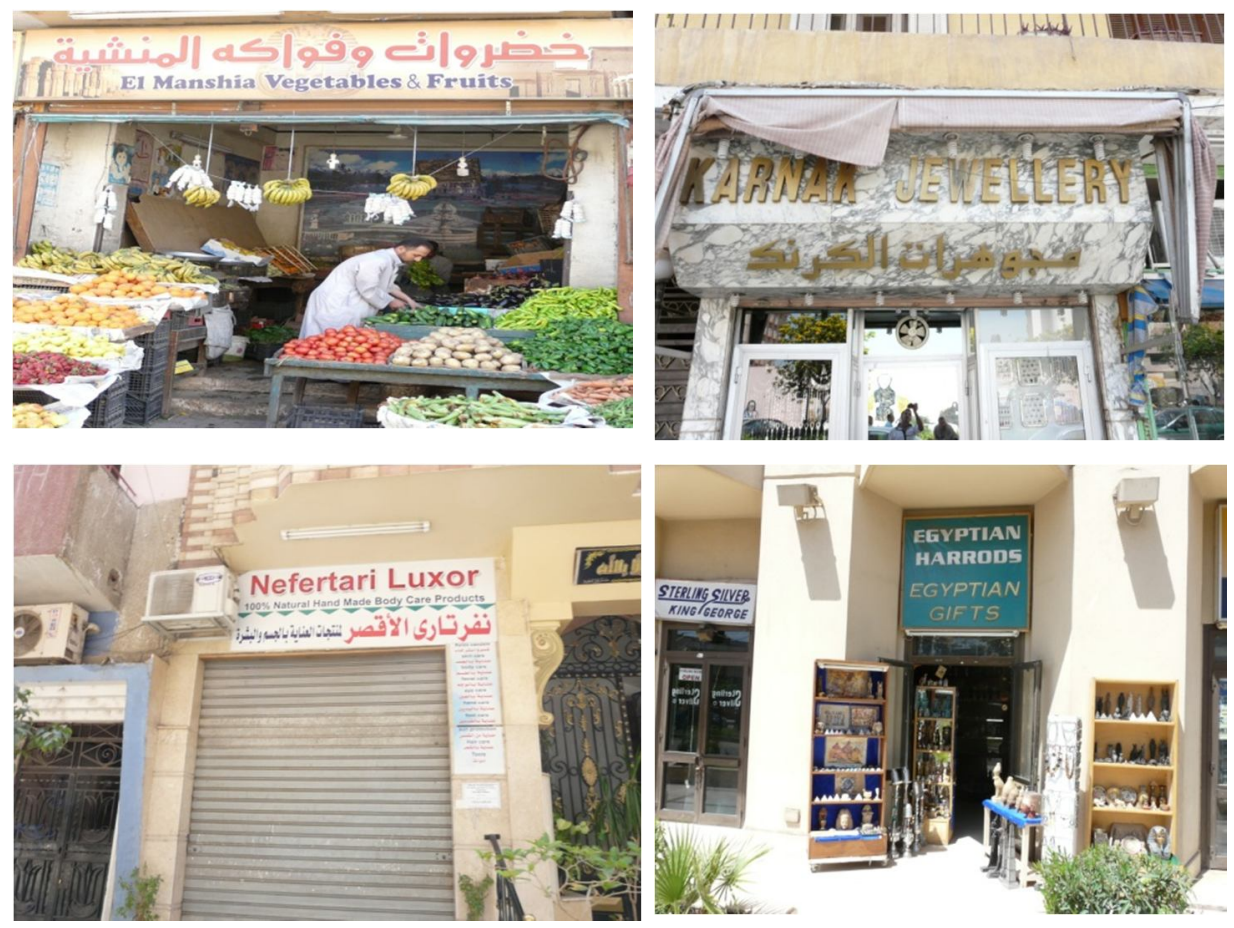

Nous remarquons que le "ici" n'est pas exprimé explicitement mais implicitement. Le locuteur évoque, sur ces enseignes, le nom du quartier où il vit comme dans la première photo Manchia, puis nous voyons un autre commerçant qui mentionne le mot Karnak qui se réfère à la ville de Louxor et ses antiquités célèbres, puis on choisit la reine de Nefertari comme titre. Ce choix est hautement symbolique puisque celle-ci est la principale grande épouse royale du pharaon Ramsès. Elle était nommée la dame de charme ou maîtresse de la haute et de la basse Egypte. Ainsi, mettre Nefertari sur une enseigne d'un magasin des accessoires de beauté signifie que le locuteur appelle les clients et leur notifie que chez lui il y a les secrets de la beauté royale et que ses produits sont originaux.

$\mathrm{Au}$ niveau de la dernière enseigne le commerçant opte pour l'emploi de l'adjectif égyptien pour appeler non seulement les passants de la rue mais aussi les touristes étrangers cherchant les objets très rares et qui veulent acheter des souvenirs touristiques de l'Egypte. Par l'emploi de ces mots exprimant l'identité géographique, le locuteur commerçant dévoile son appartenance culturelle et spatiale d'un côté, et invite ses destinataires (touristes ou égyptiens) à partager avec lui cet espace urbain qu'il s'est approprié de l'autre côté.

En somme, il apparaît en toute évidence pour nous la forte présence de déictiques de l'ailleurs dans la dénomination des magasins de la ville de Louxor. Ce qui souligne que ce n'est pas un indicateur de transition non seulement spatial mais également culturel. La définition de l'ailleurs est plus ou moins imprécise géographiquement et souvent particulièrement vague au plan culturel. Cela peut être 
une référence à un pays où à des habitants. Voici des exemples pour mieux comprendre cette idée:
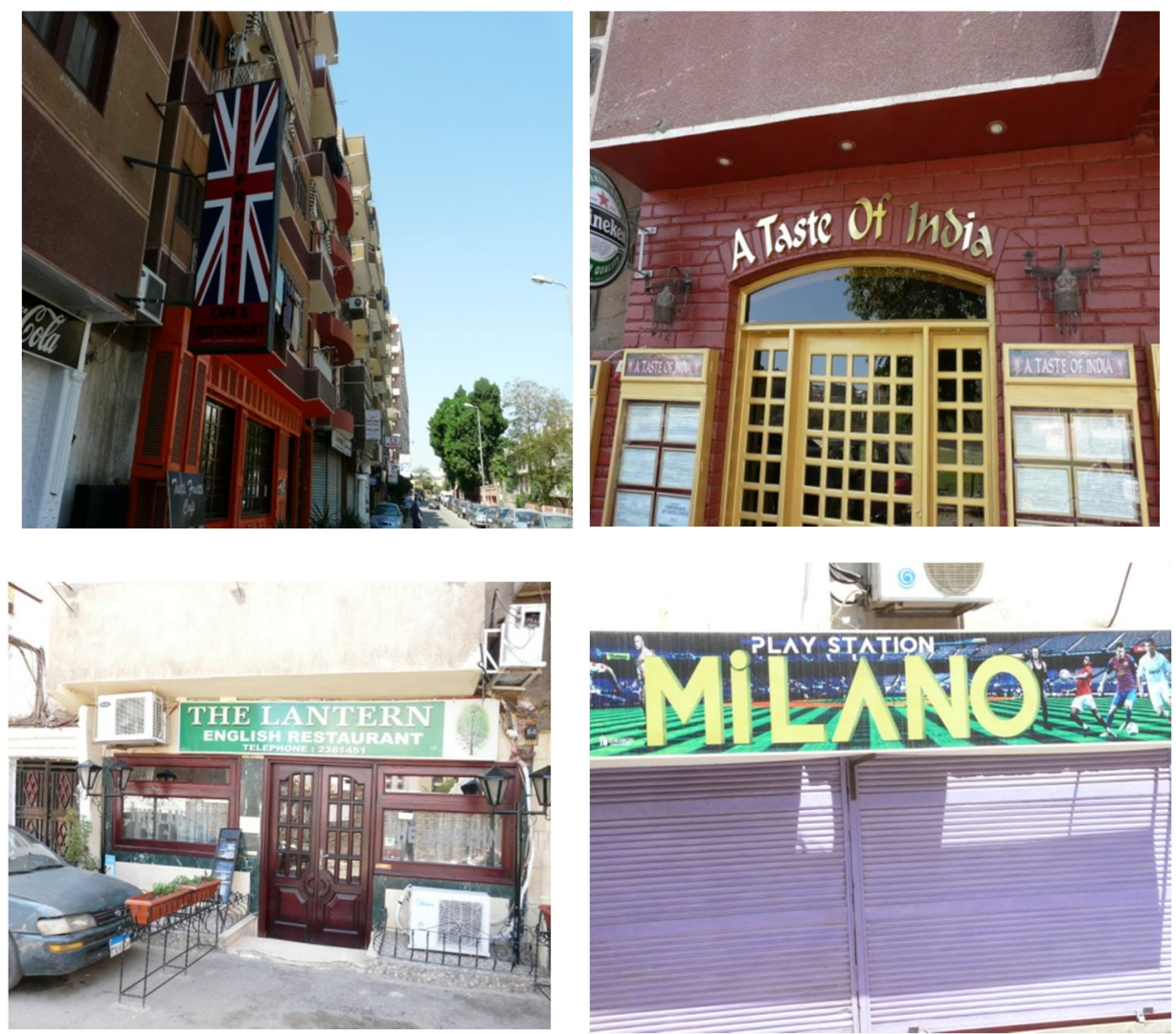

Le locuteur de la première enseigne écrit en italien le nom du restaurant et met le drapeau de l'Angleterre comme un symbole et référence spatiale de son magasin. La deuxième enseigne taste of india indique que le restaurant présente des repas indiens. La troisième est pour un magasin pour les jeux vidéo qui choisit l'équipe italienne célèbre Milano comme un titre pour attirer l'attention de ceux qui sont passionnés par les jeux de football de venir nombreux chez lui.

La quatrième annonce qu'il s' agit d'un restaurant anglais. A la question posée au propriétaire du magasin dont le titre English Restaurant (restaurant anglais) de savoir s'il présente d'autres repas au magasin et la nature des clients qui viennent chez lui, il a montré que son magasin présente de différents repas et pas seulement les plats anglais et que ses clients sont des égyptiens et des touristes. Il semble que le choix des titres occidentales pour les enseignes commerciales devient significatif puisque pour le commerçant c'est un bon moyen pour dire que son magasin est moderne et distingué et pour créer un espace exotique au sein de la ville et il essaye d'inciter les passants dans cette rue à venir déguster et consommer les délices de son restaurant. Le choix de ces titres ne convient jamais à la nature de ce qui est présenté à l'intérieur du restaurant comme ce qui parait clair dans la réponse du propriétaire du English restaurant. Donc le choix du drapeau et des mots étrangers traduit que la 
culture et la langue européenne est toujours valorisée chez un bon nombre des propriétaires des magasins dans la ville de Louxor et ces moyens les rendent toujours modernes et cultives.

\section{La référence temporelle}

D. Maingueneau souligne que "Les points de repère des indications temporelles, c'est le moment où l'énonciateur parle, le "moment d'énonciation, qui définit le présent linguistique ${ }^{27 " .}$. Dans les enseignes commerciales, nous avons découvert que certains commerçants utilisent des mots et des expressions qui évoquent des moments anciens mais dans un cadre nostalgique. Voici des exemples:

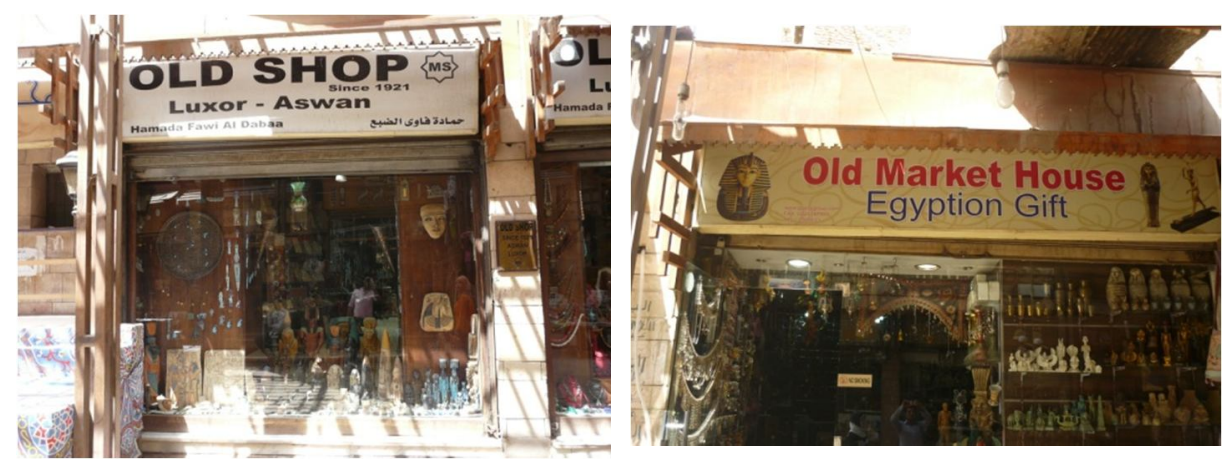

En lisant ces deux enseignes nous avons deux possibilités comme le note Mainguneau: "Deux possibilités s'offrent: soit le procès dont on entend déterminer l'origine dure encore au ME (durée ouverte), ça fait trois jours qu'il pleut sans arrêt; soit il est achevé (durée fermée) ${ }^{28}$." Dans ces deux enseignes nous observons l'emploi de l'adjectif Old (ancien) et la date de 1920 qui suit le déictique temporel since (depuis) et qui dure jusqu' à présent. Ici le locuteur utilise un repère rétrospectif d'une durée ouverte. Le but de l'emploi de la durée rétrospective ouverte chez le commerçant est d'attester l'authenticité et l'originalité des produits dans son magasin ou dans sa fabrique notamment il vend des antiquités pharaoniques pour les touristes cherchant l'originalité et les antiquités rares et bien fabriquées.

Dans d'autres magasins, nous notons la présence de l'adjectif ancien pour mémoriser du beau temps et le celui de la richesse musicale et de la gloire artistique:

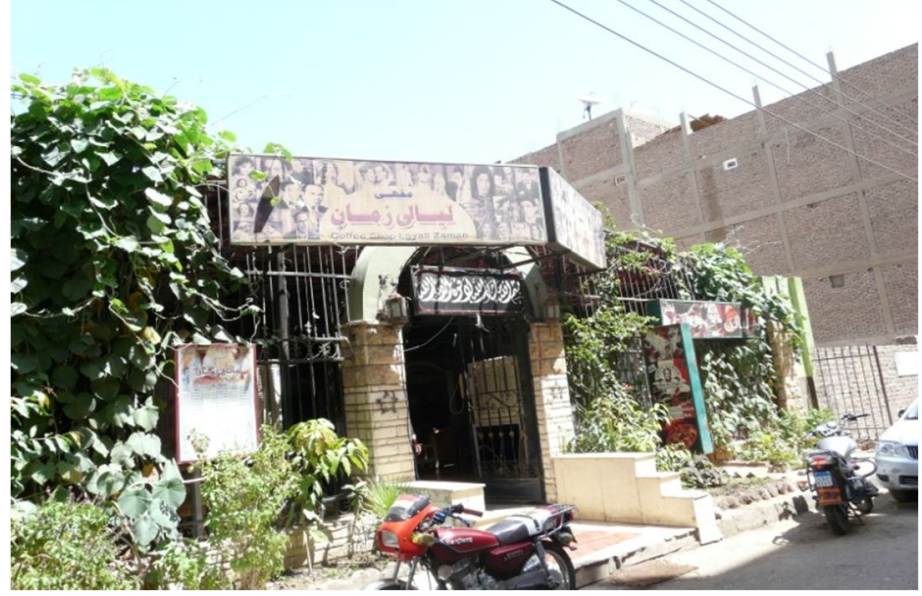


Quant à l'enseigne ci-dessus le locuteur choisit قهوة ليالى زمان (Café des anciens jours) comme titre de son café. Cette enseigne fait appel, par la mise des photos des chanteurs et des musiciens égyptiens, au destinataire pour lui rappeler le temps de la richesse de l'art musical et artistique des chanteurs et des acteurs musicaux comme Abdelhalim Hafez, Abdelwahab et Laila Mourad comme chanteurs et Farid Chawki, Abdelfattah Elkossary Naguib Elrehany ... en tant acteurs ayant hautement influencé le cinéma et le chant égyptien et arabe. Le commerçant essaye de convaincre le client ou le passant que chez lui l'on peut voir et écouter des chansons classiques et originales. L'énonciateur sait bien que tous les égyptiens adorent la musique classique qui se distingue par la belle voix et par le bon choix des mots. Donc l'élément du temps est presque fermé puisqu'il se limite à un temps déjà déterminé et le commerçant l'utilise seulement comme un moyen d'attirer les clients et ressusciter la belle époque de l'art musical arabe.

\section{La connotation et le recours au jeu des mots:}

Dans notre corpus des enseignes commerciales dans la ville de Louxor, nous avons découvert une autre spécificité dans ces écrits urbains. Nous avons constaté que les locuteurs font recours aux connotations et au jeu de mots. Ils se servent de ce type de figures pour s'identifier davantage dans cet espace urbain et pour inciter le lecteur de ces inscriptions à décoder leurs messages et interpréter ce qu'elles veulent dire. A cet égard Jean Pierre Sautot et Vincent Lucci soulignent que " pour se
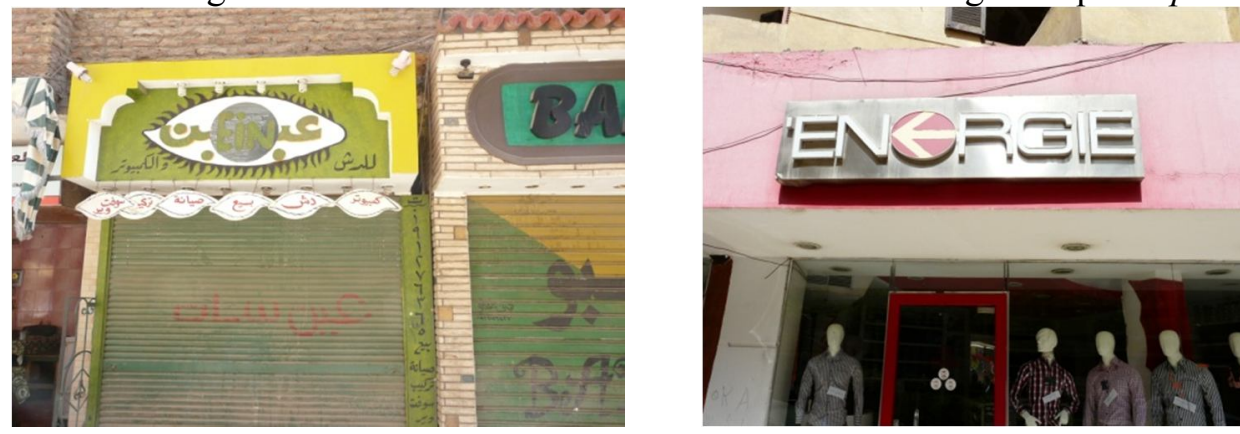

démarquer d'un usage prosaïque et ordinaire du langage, on a recours dans les noms de commerces des centres urbains à des jeux sur la langue: ils portent sur les mots eux-mêmes (incluant leurs charges connotatives) mais aussi sur les virtualités de découpages distincts à l'oral et à l'écrit sur l'orthographe, ou sur l'imbrication de la composante iconique au fonctionnement linguistique ${ }^{29}$."Voici des exemples :

Dans ces deux exemples, le locuteur joue sur le mot :

- Au niveau de la première enseigne, on peint un oeil et écrit en arabe عبن et au milieu du mot il insère en lettres latines le mot Ein.

- Au niveau de la deuxième, il écrit le mot ENERGIE et il peint la lettre $E$ à la forme d'une flèche. Pour déchiffrer les formes de la variation graphique, le lecteur doit faire appel à son répertoire culturel et linguistique et ça dépend bien sur du niveau éducatif de son apprentissage:

"Le rapport que tisse le lecteur avec le système d'écriture tient aux conditions familiales et scolaires de son apprentissage. La sensibilité à la variation 
orthographique présentée dans certaines enseignes est assujettie à la subjectivité du lecteur (...)30."

Donc nous comprenons que le décodage des mots appelle à l'expérience du lecteur et son niveau culturel et langagier. Bien plus, Si le lecteur n'accède pas à décoder la nature de l'écriture de l'enseigne commerciale, il va les percevoir comme anormales et incompréhensibles. Nous pouvons dire que le jeu sur les mots dans l'enseigne commerciale peut être utile sur le niveau de la fréquentation du magasin et sur le niveau d'achat, puisque cette forme étrange va hanter la mémoire du lecteur et du passant pour toujours et l'invite à voir l'activité commerciale sur place :

" En effet, la fréquentation du commerce dissipe la méfiance initiale. En marquant durablement la mémoire du lecteur, et malgré une première opinion négative, la figure orthographique y installe l'identité du commerce 31 ".

Nous constatons que la connotation se fait également par l'emploi des enseignes bilingues ou trilingues. C'est pour marquer que le propriétaire du magasin est cultivé et qu'il possède presque une réputation prestigieuse. Voici deux exemples:

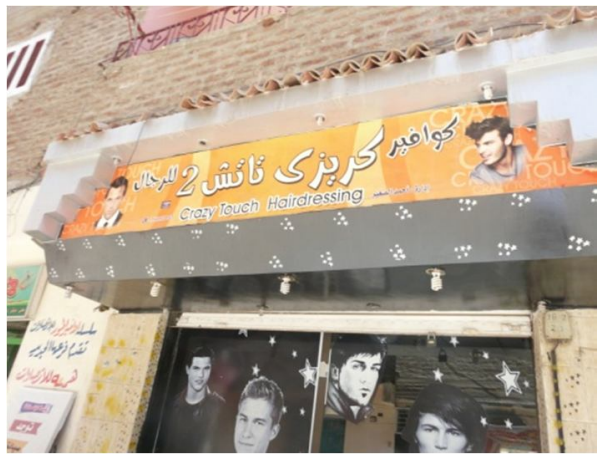

Rue de la télévision

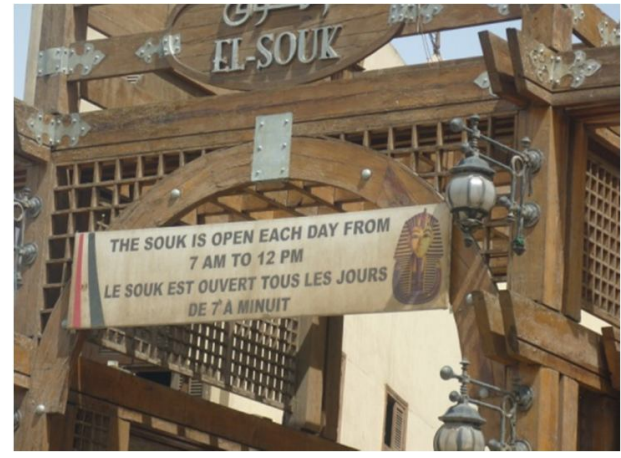

Rue du marché touristique

Le locuteur dans la première enseigne utilise le français (coiffeur) en caractères arabe, l'anglais (Crazy Touch) et l'arabe ( للرجال pour les hommes) et dans la deuxième enseigne, on utilise une phrase complète en anglais et sa traduction vers le français. Nous pouvons indiquer que le commerçant utilise d'autres langues comme l'italien et le coréen et d'autres langues. Le commerçant essaye d'utiliser de nouvelles formes et de nouvelles structures d'écriture pour se montrer moderne et ouvert sur le monde entier. Bref, le recours à ces différents types de connotations vise à personnaliser le commerce et à partager avec les clients ou bien les passants les représentations culturelles et spatiales renvoyant à ces espaces urbains en circulation et en mouvement permanents.

\section{Le recours à l'hyperbole}

L'hyperbole accentue l'expression d'une idée par rapport à sa réalité; c'est à dire, c'est une exagération. Nous pouvons trouver quelques enseignes qui utilisent cette forme : 


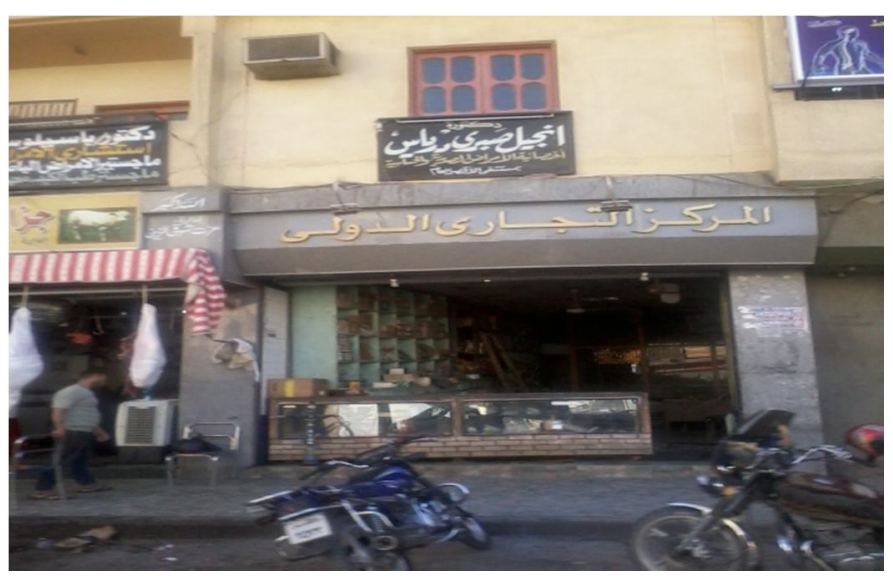

Rue Abou Elgoud

Nous observons ici que le commerçant choisit المركز التجارى الدولى (le centre du commerce international) comme formulation des inscriptions sur une enseigne de son petit magasin qui vend les accessoires des motos. Le locuteur choisit ce titre à l'exemple du CCI (centre du commerce international), aux Etats-Unis, qui est une agence conjointe de l'organisation mondiale du commerce et de l'organisation des Nations unies fondée en 1964. Le choix du titre et l'activité pratiquée dans le magasin ne sont pas symétriques mais cela traduit évidemment que les commerçants utilisent tous les moyens possibles pour attirer l'attention des clients même si le choix du mot est exagéré et pas logique. D'ailleurs le propriétaire du magasin recourt parfois à l'emploi du superlatif ${ }^{32}$ pour marquer sa singularité. Voici un exemple:

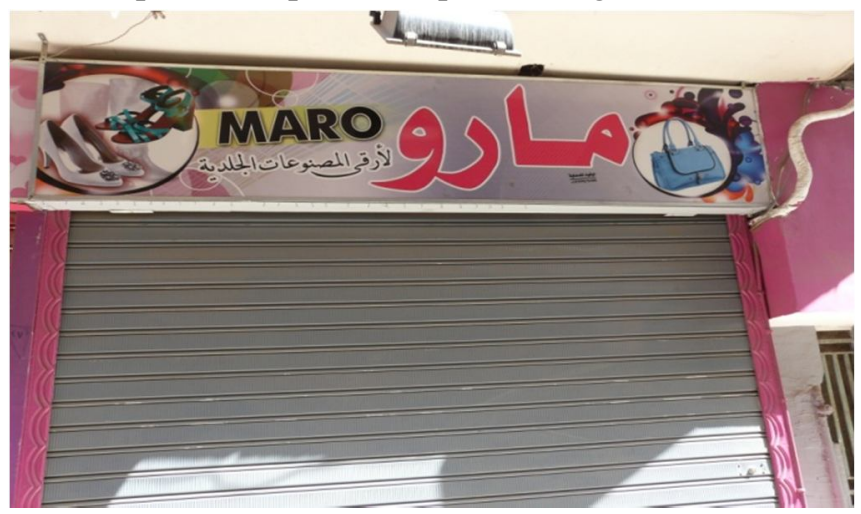

place de la gare

Dans l'enseigne ci- dessus, le locuteur du magasin commercial choisit l'adjectif ارقى الهصنوعات الجلدية(le meilleur dans l'industrie du cuir). Cet adjectif indique que le commerçant présente la bonne qualité et les bons produits. C'est un type de propagande qui pousse le client à venir chez lui. Mais nous pouvons dire que le vendeur perd souvent la crédibilité dans ce cas parce que les commerçants utilisent souvent le même adjectif mais avec le changement de l'adjectif par le plus beau, le plus prestigieux... ou en préférant parfois le terme «numéro 1 », ou le «premier en ....» Donc le locuteur utilise l'hyperbole pour convaincre son client et fait augmenter le nombre de ses visiteurs. 


\section{Conclusion:}

De tout ce qui précède, nous comprenons que l'enseigne commerciale représente un bon moyen pour attirer l'attention du client et l'inciter à visiter le magasin sur place. Nous avons constaté que les commerçants en tant que sujet d'énonciation expriment leurs spécificités plurilinguistiques de l'écriture de l'enseigne par plusieurs manières. Ils se dévoilent à travers les langues dont ils se servent et à travers les discours qu'ils tiennent. Les enseignes commerciales deviennent un bon moyen pour le propriétaire du magasin de s'identifier et s'imposer dans le milieu citadin. La ville de Louxor devient presque une scène dans laquelle la concurrence touristique se traduit par la concurrence langagière. Nous avons découvert que le choix de la langue dans l'enseigne commerciale à Louxor n'a pas de règles fixes et chaque commerçant a sa stratégie sans se restreindre aux lois de l'écriture et chacun se croit la porte parole de la modernité et de l'originalité dans l'écriture de l'enseigne. L'emploi de la langue étrangère aux yeux d'un grand nombre de commerçants traduit la culture et la modernité. Chez un nombre restreint de commerçants l'arabe est une langue sacrée qui est capable de s'imposer dans le marché langagier. Nous avons compris que l'effet de l'urbanisation et du tourisme dans la ville de Louxor a sa contrepartie sur l'appropriation des espaces et la diffusion des langues. Nous avons estimé que la ville de Louxor représente un lieu d'expression et d'une histoire, d'une culture et d'une identité. Les enseignes commerciales témoignent d'une hiérarchisation socio- spatiale accompagnée par effet d'une stratification sociale des langues en présence dans cet espace urbain. Grosso modo, l'étude des enseignes commerciales a permis de découvrir et de saisir la situation plurilingue dans les villes touristiques en Egypte en général et la ville de Louxor en particulier. 


\section{Bibliographie:}

1 - Ouvrages d'ordre linguistique

- Agnès MILLET, La ville, un espace socio- sémitique, dans des écrits dans la ville. Sociolinguistique d'écrits urbains: l'exemple de Grenoble, l'Harmattan, Paris, 1993.

- Dominique MAINGUENEAU, l'énonciation en linguistique française, Paris, Hachette, 1999.

- Emile GINOT, Les enseignes: leur origine et leur rôle, BNF, 1901.

- Henri VERDIER , La publicité, Que Sais-je?, Paris, P.U.F, 1957.

- J. CALVET, La guerre des langues et les politiques linguistiques, Payot et Rivages, Paris, 1987

- J. CALVET, Les voix de la ville: introduction à la sociolinguistique urbaine, Payot, Paris, 1994.

- Kerbat ORCCHIONI, l'énonciation, Paris, Armand Colin, 1980.

- Maurice GREVISSE et André GOOSSE, Le bon usage Grammaire française, 14 éditions, Bruxelles, Editions de Boeks Universités, 2008.

- Olivier SOUTET, Linguistique, Paris, P.U.F. 2eme édition, 1997

- P. Charaudeau, Langage et discours, éléments de sémio linguistique. Paris Hachette, 1983.

- R. GALISSON, De la langue à la culture par les mots. Paris : Clé Internationale [coll. R. Galisson], 1991.

- Safia ASSALAH-RAHAL, Plurilinguisme et migration, L'Harmattan, Paris, 2004.

- V. LUCCI, En quête d'une identité, Des écrits dans la ville : sociolinguistique d'écrits urbains : l'exemple de Grenoble, Paris, L'Harmattan, 1998.

- Constitution de la république arabe d'Egypte 2014 ستور جمهورية مصر العربية'

\section{2-Articles de revues, de journaux et de colloques}

- A. BOUDREAU, L'affichage à Moncton : miroir ou masque ? dans Signalétique langagière et linguistique des espaces de ville (configuration et enjeux sociolinguistiques), revue de l'université de Moncton, vol. 36, $\mathrm{n}^{\circ} 1$, Nouveau Brunswick (Canada), 2005, p. p. 185-217.

- Caroline JULLIARD, le plurilinguisme, objet de la sociolinguistique, revue Langage et société 2007/3-4 (numéro 121-122), p.336.

- F. BOUMEDINE, Etude sociolinguistique des quartiers de la ville de TiziOuzou: identité urbaine et territoriale dans Ségrégation spatio-linguistique et Dynamiques socio-langagières et habitat dit populaire, Atfalona-DKA, Alger, 2007, p.p. 207231.

- F., LEIMDORFER, Des villes, des mots, des discours, dans langage et société, $\mathrm{n}^{\circ}$ 14, Maison des sciences de l'homme, Paris, 2005.

- Fodil Mohammed SADEK, Reconnaissance linguistique et construction identitaire en Algérie: ce que nous disent les enseignes commerciales en anglais à Tiziouzou, Colloque International organisé par l'IRCAM, Rabat, Maroc, du 20 au 21 Novembre 2014.

- Jean-Pierre SAUTOT, Vincent LUCCI, Lire dans l'espace urbain, les paradoxes des enseignes commerciales, Revue Langage et société, N 96, juin 2001.

- Pierre Bouchard, enquête sur la langue de l'affichage commercial sur l'île de Montréal en 2010, Bibliothèque et archives nationales de Québec, 2012. 
- Romain LAJARGE, Claudine MOISE, Enseignes commerciales, traces et transition urbain. Quartier de Figuerolles, Montpellier, Revue de l'université de Moncton, Vol 36, N 12005.

- R. NOGUES, Fonctions du patronyme et effet dans la clinique. Le trimestre psychanalytique, Paris, Editions de l'association freudienne, 1.23-30, 1992,

\section{3- Dictionnaires}

- Grand dictionnaire universel du XIXe siècle, 1870.

- J. DUBOIS, et all., Dictionnaire linguistique et des sciences du langage, LarousseBordas/HER, Paris, 1999.

- P. CHARAUDEAU, Dictionnaire d'analyse du discours, Seuil, Paris, 2002.

- Dictionnaire encyclopédique, culture générale, langue française, Larousse (VUEF), Paris-Cedex, 2002.

- Le Nouveau Petit Robert de la langue française, 2010.

\section{4- Thèses}

- Zohra BENMNSOUR, Marquage signalétique plurilingue et appropriation de l'espace urbain, Université de Batna, Algérie, 2010,

- Tigziri Noura, Les enseignes en français dans trois contextes sociolinguistiquesurbains:Lausanne, Alger,Tizi-ouzou.

https://www.google.dz/search?q=Les+enseignes + en + fran $\% \mathrm{C} 3 \% \mathrm{~A} 7 \mathrm{ais}+$ dans + trois + contextes + sociolinguistiques + urbains $\% 3 \mathrm{~A}+$ Lausanne $\% 2 \mathrm{C}+\mathrm{Alger} \% 2 \mathrm{C}+$ Tizi-

Ouzou. 


\section{Les références:}

1 Caroline JULLIARD, le plurilinguisme, objet de la sociolinguistique, revue Langage et société 2007/3-4 (No 121-122), p.336.

2 Romain LAJARGE, Claudine MOISE, Enseignes commerciales, traces et transition urbain. Quartier de Figuerolles, Montpellier, Revue de l'université de Moncton, Vol 36, N 12005 , p.99.

3 Jean CALVET, Les voix de la ville: introduction à la sociolinguistique urbaine, Payot, Paris, 1994, P. 130.

4 Caroline JULLIARD, Op. Cit., P. 338

5 Grand dictionnaire universel du XIXe siècle, 1870, p. 134

6 Emile GINOT, Les enseignes: leur origine et leur rôle, BNF, 1901, P.7

7 Loc. Cit

8 Le Nouveau Petit Robert de la langue française 2010, p. 882.

9 Cf., Zohra BENMNSOUR, marquage signalétique plurilingue et appropriation de l'espace urbain, Université de Batna, Algérie, 2010, P. 26

10 Agnès MILLET, la ville, un espace socio- sémitique, dans des écrits dans la ville. Sociolinguistique d'écrits urbains: l'exemple de Grenoble, l'Harmattan, Paris, 1993, p. 26.

11 Zohra BENMNSOUR, Op. Cit., P. 29

12 "La politique linguistique comme l'ensemble des choix conscients effectués dans les domaines des rapports entre langue et vie sociale et plus particulièrement entre langue et vie nationale, et une planification linguistique, comme la recherche et la mise en ouvre des moyens nécessaires à l'application d'une politique linguistique" J. CALVET, la guerre des langues et les politiques linguistiques, Payot et Rivages, Paris, 1987, P. $\overline{55 . \text { Nous }}$ pouvons dire qu'en Egypte l'anglais représente parfois la première langue étrangère en concurrence avec le français. Parfois nous observons les autres langues comme l'allemand, l'italien et l'espagnol comme deuxième langue étrangère dans l'étape secondaire.

13 Constitution de la république arabe d'Egypte 2014

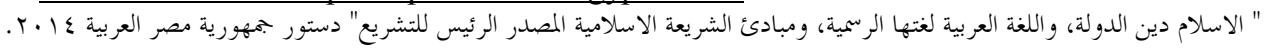

14 H.VERDIER, La publicité, Que Sais-je?, Paris, P.U.F, P.74.

15 Dominique MAINGUENEAU, l'énonciation en linguistique française, Paris, Hachette, 1999 , p. 9

16 Patrick CHARAUDEAU, Dictionnaire d'analyse du discours, Paris, Seuil, 2002, P. 228.

17 Verdier H, Op. Cit., P. 134

18 Romain Lajarge et Claudine Moise, Op. Cit., P. 108.

19 Kerbat ORCCHIONI, l'énonciation, Paris, Armand Colin, P. 23.

20 Romain LAJARGEet Claudine MOISE, Op. Cit., P. 109

21Cf., Maurice GREVISSE et André GOOSSE, Le bon usage Grammaire française, 14 éditions, Bruxelles, Editions de Boeks Universités, 2008, p.462.

22 Le patronyme est le nom de famille formé après le nom du père, soit directement (en France, Dupont, Durand, etc.), soit sous la forme de dérivé (fils de Jean, etc.). Jean DUBOIS et ALII, Dictionnaire de linguistique, Paris, Larousse, 2002, P. 353.

23 R. NOGUES, Fonctions du patronyme et effet dans la clinique. Le trimestre psychanalytique, Paris, Editions de l'association freudienne, 1.23-30, 1992, P. 28

24 Romain LAJARGE, Claudine MOISE, Op. Cit., P. 114.

25 Dominique MAINGUENEAU, Op. Cit., P. 35

26 Ibid, P.35: 36

27 Dominique MAINGUENEAU, Op. Cit., P. 36.

28 Ibid., P. 41.

29 Jean-Pierre SAUTOT, Vincent LUCCI, Lire dans l'espace urbain, les paradoxes des enseignes commerciales, Revue Langage et société, N: 96, juin 2001, p. 31

30 Ibid., P.36, 37

31 Ibid, 39.

32 le superlatif est le degré de la comparaison de l'adjectif ou de l'adverbe exprimant la qualité ou la modalité à un degré très élevé, supérieure ou inférieure à d'autres ou indépendamment de toute référence. Cf. Maurice GREVISSE et André GOOSSE, Op.Cit., P. 510. 